\title{
Mining usage data for Adaptive Personalisation of Smartphone based Help-on-Demand services
}

\author{
William Burns \\ Computer Science Research Institute Computer Science Research Institute \\ University of Ulster \\ United Kingdom \\ +44 (0)2890368918 \\ wp.burns@ulster.ac.uk \\ Mark Donnelly \\ Computer Science Research Institute Computer Science Research Institute \\ University of Ulster \\ United Kingdom \\ +44 (0)2890366330 \\ mp.donnelly@ulster.ac.uk \\ University of Ulster \\ United Kingdom \\ +44 (0)2890368837 \\ I.chen@ulster.ac.uk \\ Kerry Louise Skillen \\ University of Ulster \\ United Kingdom \\ +442890368918 \\ kl-skillen@email.ulster.ac.uk
}

\author{
Chris Nugent \\ Computer Science Research Institute \\ University of Ulster \\ United Kingdom \\ +44 (0)2890368330 \\ cd.nugent@ulster.ac.uk \\ Ivar Solheim \\ Norwegian Computing Centre \\ Oslo \\ Norway \\ ivar.solheim@nr.no
}

\begin{abstract}
Mobile computing devices and their applications that encompass context aware components are becoming increasingly more prevalent. The context-awareness of these types of applications typically focuses on the services offered. In this paper we describe a framework that supports the monitoring and analysis of mobile application usage patterns with the goal of updating user models for adaptive services and user interface personalisation. This paper focuses on two aspects of the framework. The first is the modelling and storage of the usage data. The second focuses on the data mining component of the framework, outlining the five different capabilities of the adaptation in addition to the algorithms used. The proposed framework has been evaluated through specific case studies, with the results attained demonstrating the effectiveness of the data mining capabilities and in particular the adaptation of the User Interface. The accuracy and efficiency of the algorithms used are also evaluated with three users. The results of the evaluation show that the aims of the data mining component were achieved with the personalisation and adaptation of content and user interface, respectively.
\end{abstract}

\section{Categories and Subject Descriptors}

H.3.3 Information Search and Retrieval, clustering, data mining, recommendation.

\section{General Terms}

Algorithms, Usage Patterns, Data Mining, Personalisation, Design, Human Factors.

\section{Keywords}

Usage Mining, Clustering, Adaptation, Personalisation, Smartphone Applications.

\section{INTRODUCTION}

An ageing society is being established as a result of increased life expectancy and falling birth rates [1]. A further result of these trends is the increased burden being placed on social and health care services. As a result, an increasing focus is being placed on using ICT (Information and Communication Technology) as a possible means to alleviate these burdens.
It is, however, important that any solution offered by the use of ICT be personalised to the end user's physical, cognitive and age related requirements. Any developed solution is less likely to have a positive effect on the user's quality of life if it does not address the user's requirements and physical capabilities [2]. Nevertheless, there is a challenge in ascertaining a user's context primarily based on their interaction with the technology. The data captured during, what may be, brief interactions with a device needs to be designed in such a way to contain as much physical in addition to application/service context based information that can be retrieved at a later point in time [3].

This paper presents a framework that aims to collect usage data from a Smartphone based application and extract usage patterns using rule based and clustering algorithms for the purposes of providing personalised and adaptive content and user experience.

In Section 2 we outline related work in the area of mobile assistive technologies and the tools and methods in which usage data is used for the purposes of personalisation. Section 3 presents a general overview of the proposed framework that aims to provide Help-on-Demand and context-aware support to older Smartphone users. This is followed by Sections detailing the collection and storage of the usage data, the algorithms used for data mining, a usage scenario and results of an evaluation of the algorithms used.

\section{RELATED WORK}

Utilising mobile technologies to provide assistive technology solutions, such as Smartphones and tablet devices, afford end users the potential to benefit from the associated assistive services as they move within and between environments [4]. The increased mobility, coupled with the in-built hardware such as GPS modules, accelerometers and gyroscopes in addition to wireless communications offer the potential to infer a user's current state in terms of their current context and situation. A system is said to be context aware "... if it uses context to provide relevant information and/or services to the user, where relevancy depends on the user's task." [5].

In recent years a number of studies have used mobile technologies in order to provide a technological solution to a number of challenges and unmet needs that are associated with old age. In 
the area of Alzheimer's care, a number of projects have aimed to provide persons with dementia with navigational assistance using a smartphone with in-built GPS hardware [6]. Also investigated has been the user of mobile phone technology to provide video reminders to persons with mild dementia [7]. With both of these solutions the notion of a virtual caregiver is created.

Due to the connectivity of Smartphones, peripheral devices such as keypads, mice and sensors can be added to the technological solution to provide a better user experience and service. One example is a solution that used wireless sensors in conjunction with a Smartphone to record and monitor the user's heart and respiration rates in addition to their activity levels [8]. These solutions aim to provide support for physical and cognitive disabilities, however, the services offered are not personalised to the end user's behavioural patterns. This leads to a reduced provision of context aware information and services.

Context aware applications tend to either focus on the modeling of static user characteristics such as GUMO [9] or alternatively UPOS [10], which focuses primarily on the dynamic situations surrounding the user, however, such solutions fail to offer a comprehensive user profile or personalisation or adaptation in any detail. Context aware service adaptation not only needs to consider the user's context, but also their preferences, and hence support both the personalisation and the context aware features [11].

In order to provide a personalised solution, the system must understand the needs and usage patterns of the user during the lifetime of the system. To achieve this, a log of user interactions within the application is required. Previous work in Smartphone data mining has focused on using GPS to establish 'stay areas' [12], and accelerometer data to establish physical activity recognition [13], such as walking, walking up stairs or sitting, for example. All of this information enables the provision of an assistive system that will be more aware of the user's physical context.

To date, relatively few studies have addressed issues associated with personalisation and adaptation of both content and User Interface (UI). One such study [14] aims to address these areas in order to provide personalised and adaptive content to tourists via a Smartphone. This system updates the UI based on web technologies and offers adaptation based on a user profile and context only. Due to the short-term use of the Smartphone, i.e. duration of holiday, there is no storage of usage patterns that would enable the user profile and adaptation services to evolve.

In this paper we propose a framework that aims to address the issue of providing an adaptive application UI and content, based on a personalised user profile. In the following Section we introduce our framework to address the issue of personalisation and adaptation of a technological system based on user interaction patterns and user profiles.

\section{ADAPTATION FRAMEWORK}

MobileSage is an EU Ambient Assisted Living funded project [15], which aims to provide older persons with context-sensitive personalised and location sensitive tools to allow them to carry out and solve everyday tasks and problems. There are two main components of this system as presented in Figure 1. The first is a Smartphone based application, called Help-on-Demand (HoD). The HoD offers three key services, Search, Scan NFC/QR and Travel (Directions and Search places of interest) and emergency contact service. A help facility is included that will help the user use the services efficiently.

The second component is a Content Management System (CMS). The HoD allows the user to access content on an 'as needed' basis that helps them accomplish their everyday tasks. This content ranges from instructions on how to use everyday household appliances towards engaging with more involved activities such as completing the purchase of a ticket from a machine at a train station. Additionally, the system provides location specific content such as the directions between two places or towards a point of interest. The CMS contains all of the information that would be requested from the user. The content comes in a number of modalities, such as text, audio and video that are prioritised based on the user's User Profile.

The Adaptation Framework [16] contains four core services, Personalisation, Profile and Context in addition to a Dialog Manager, that serves as a part of the HoD application and allows the services, content and UI of the system to be adapted and persoanlised based on the user's usage patterns and profile. The Adaptation Framework, as presented in Figure 1, is a component of the HoD application and is used to collect usage data, personalise content, ascertain the user's context and manage requests and responses to and from the CMS. The adaptation framework allows the HoD application to evolve and adapt to the usage patterns of the user.

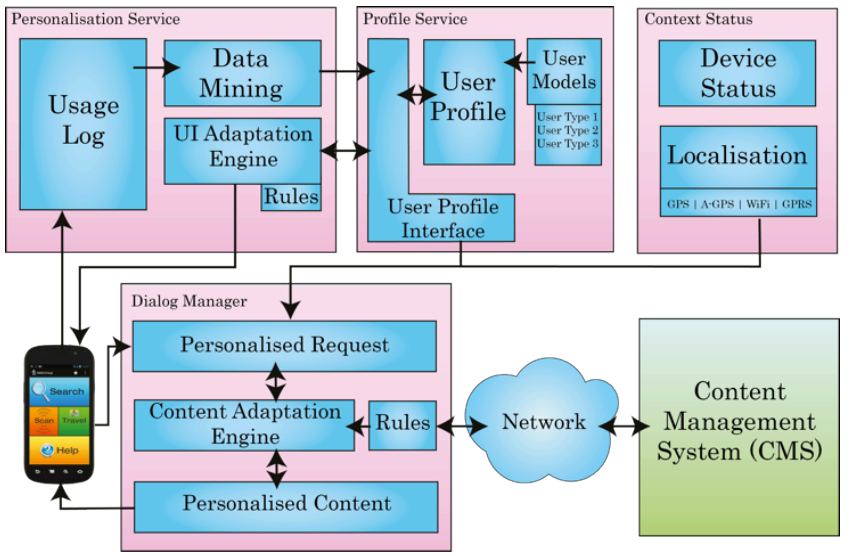

Figure 1. Information flow within the Help-on-Demand application using the proposed Framework. Four core services exist to provide information to the user and to personalise requests to the Content Management System. (This paper addresses the Usage Log, Data Mining and UI Adaptation Engine components within the overall framework.)

The User Profile is generated during the initialisation phase of the HoD. It is based on one of three User Models: Technophobic, Techno-Static and Technophile and outlines the parameters contained within the User Profile, based on the type of user. Each of these User Models generates User Profiles with differing levels of complexity. Technophobic should offer core functionality and require as little cognitive load and user interaction as possible by limiting the amount of user initiated personalisation i.e. selection of positioning modules or services. The Technophile model will offer the greatest complexity settings and could be considered an advanced User Profile. The Techno-Static model would be an amalgamation of the previous two.

We have developed a proof of concept application that mimics the services and UI offered as part of the MobileSage HoD 
application, which is still in the development stages. Specifically, the current paper focuses on the generation and mining of usage data and the adaptation and personalisation of content and services that will eventually be integrated into the HoD prototype. In the following sections we will focus on the 'Personalisation Service' (shown in upper-left on Figure 1) and on its three distinct components: Usage Log, Data Mining and UI Adaptation Engine.

\section{USAGE ANALYSIS AND MODELLING}

In this Section we present a usage analysis of the HoD application in addition to outlining the user data modeling process to address the scenarios presented.

\subsection{Usage Analysis}

John is a retired 68 year old male who lives in a village just outside of Belfast in Northern Ireland. He likes to go walking in the countryside and have dinner and go shopping in the city centre as often as he can. John has the MobileSage HoD application on his Smartpohone to provide him with context aware information and content. When John goes for his regular walks, he can use the HoD application to seek help or search the Travel service for directions to well-known places of interest in the countryside. For example, when John and his wife travel to Belfast via train to go shopping he uses the Scan NFC service on the HoD application to obtain instructions on how to use the electronic ticket kiosk.

The HoD application collects all of John's interactions with the application and after sufficient data is collected the system can personalise and adapt the application and content. Given that John knows where he is going when in the city center and only uses the Travel service when he is near his home village, the HoD application can listen for John's location and update the UI when he is in Belfast and remove the Travel service from the screen. This service returns when John reaches his village.

As John only uses the Scan NFC function at the train station, this too only appears when he is within a pre-defined distance to the train station. As he rarely uses the Scan QR Code service, this is removed from the UI. All services are, however, available through the use of sub-menus, if not displayed on the homescreen.

When John is out on one of his walks and requests content, the application checks the current status of the network connectivity and battery. Due to a limited data signal the system asks John whether he would like to change his preferred feedback mode to something that requires less bandwidth such as text.

John appreciates that he does not have to spend time going through a number of settings in order to personalise his application, however, simply has to use the system in order to personalise it. He also likes that the application offers the most appropriate services in the right locations and contexts making his interactions with the application much easier.

\subsection{Usage Data Modeling}

In order to monitor the user's usage patterns of the HoD, each interaction and event must be stored for retrieval by the Data Mining component. Given that the HoD application would be used on an as required basis, there is no recording of any information when the application is idle or executed in the background. This supports a level of privacy, as the user context is not ascertained without their consent, i.e. the direct use of the application.

There are four levels of interaction that are to be recorded. Level One records all general 'User Interactions'. These include deviceEvents and UIEvents. deviceEvents encompass any user interaction with the hardware of the Smartphone, such as Volume, Back and Menu button presses. UIEvents are events in which the user has interacted with the UI of the HoD. This does not necessarily mean the user has used the service they have accessed, however, merely just informs the Personalisation Service that they have moved a level through the UI.

In order to monitor the usage of the services offered within the HoD application Level Two monitors 'Service Interactions'. These events record what service the user has accessed and also what information or user input was requested. For example, if the user enters the Search service and searches for restaurants, the Usage Log would record this as a serviceEvent, including what service was accessed, in this instance Search and what the user's input was, i.e. "restaurants".

One of the core components of the HoD application is the personalisation of the services and UI. In order for this to be achieved, the user's preferences need to be stored. A part of the 'Profile Service' in the Adaptation Framework, is a User Profile. This user profile contains a number of parameters that enable the application's UI and services to be personalised. Level Three of the Usage Log involves recording the user's interaction with their User Profile, referred to as 'Profile Interactions'. All profileEvents are recorded with the profile parameter being amended in addition to that parameter's new value. For example, the user modifies the screen brightness, this would be logged as a profileEvent with the identifier 'System_Profile_Brightness' and the new value, i.e. brightness level.

The final level of event capture involves communication with the CMS. Level Four collects data in the context of 'Server Response'. When a service is accessed and a request for content is made to the CMS the request and a summary of the CMS response is recorded. These events summarise the service requesting content in addition to the user input. While the exact contents of the response can be recorded, a summary of the response is recorded. This involves what modality was returned.

All usage data is stored locally on the device itself in an SQLite Database. This allows for the querying and structuring of the data to be as simple as possible. Given that the usage data is stored on the device, it allows quick access and improved security, as no personal or sensitive information is transmitted from the device, either to the CMS or any other party.

The first two columns of the Usage Log contain the information required to ascertain the level of event accessed as presented in Figure 2. These values are used to mine the Usage Log for particular patterns relating to the Levels of interaction.

\begin{tabular}{|r|c|c|l|}
\hline eventType & serviceDetails & userInput & \\
\hline uiEvent & Service_Activation & Scan & $\begin{array}{l}\text { Level 1 } \\
\text { User Interaction } \\
\text { Level 2 }\end{array}$ \\
\hline serviceEvent & Scan_NFC & e93748264 & $\begin{array}{l}\text { Service Interaction } \\
\text { Level 3 }\end{array}$ \\
\hline profileEvent & System_Profile_Brightness & 75 & $\begin{array}{l}\text { Profile Interactions } \\
\text { Level 4 } \\
\text { System Response }\end{array}$ \\
\hline serverComm & Scan_NFC_Request_Video & e93748264 & \\
\hline
\end{tabular}

Figure 2. An example data set, and interaction levels, of a user using the Scan service within the HoD. The subsequent events show that the user modified the screen brightness, accessed the NFC option and a request was made to the CMS. 
There are five eventTypes currently supported within the Usage Log. For each, there are a number of other parameters that are used to identify each interaction a user performs. Figure 3 shows the current eventTypes and their possible serviceDetails parameters.

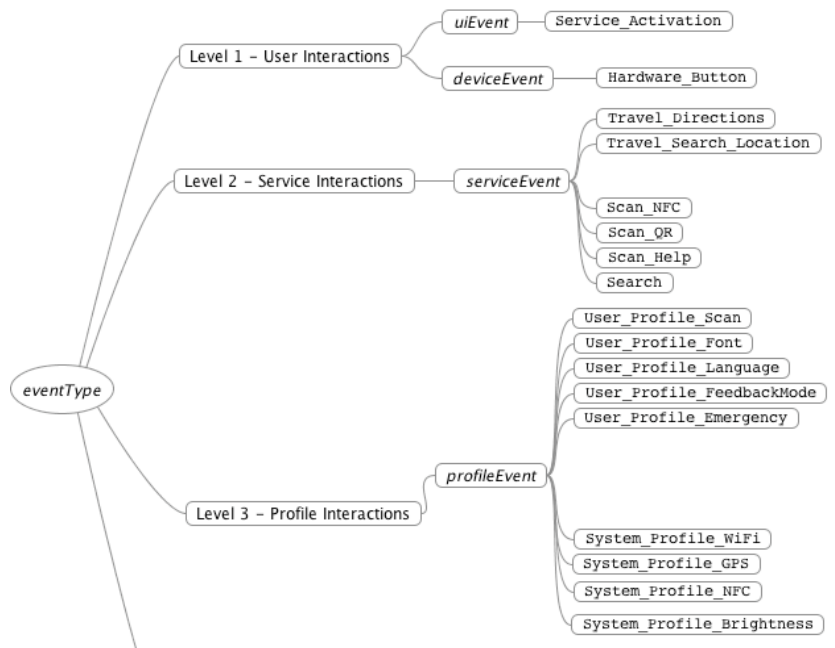

Figure 3. EventTypes and constituent serviceDetails parameters of three levels of user interaction that is to be stored in the Usage Log. These parameters will be used to identify and extract usage patterns for adaptation and personalisation.

In addition to the eventType, serviceDetails and userInput data, contextual information is also logged when any information is recorded in the Usage Log as presented in Table 1.

TABLE 1. ADDITIONAL CONTEXTUAL PARAMETERS AND EXAMPLE DATA, TO BE STORED DURING ALL USER INTERACTIONS WITH THE APPLICATION IN ADDITION TO THE EVENTTYPE, SERVICEDETAILS AND USERINPUT FIELDS.

\begin{tabular}{lc}
\hline \multicolumn{1}{c}{ Parameter } & Example Data \\
\hline The user's location (Lat \& Lon) & -5.6482742, \\
& 54.827482 \\
$\begin{array}{l}\text { What hardware is being used to obtain } \\
\text { the locations (GPS or Network) }\end{array}$ & network \\
$\begin{array}{l}\text { The connection to any Bluetooth } \\
\text { devices }\end{array}$ & $21: 74: 63: \mathrm{DE}: 1$ \\
WiFi SSID & wifiNetwork \\
WiFi Signal strength (\%) & 64 \\
GSM signal strength (\%) & 56 \\
Battery level (\%) & 89 \\
Timestamp & $2013-01-14$ \\
\end{tabular}

\section{USAGE PATTERN DISCOVERY}

In order to provide personalised services to the end user a sufficiently large dataset is required in order to extract usage patterns. Within the HoD application data mining will be performed at regular intervals to ensure an up-to-date list of services, features and UI.
The capabilities of the data mining component of the Framework address: service usage, the storage of favourite queries, the modification of requests based on device capabilities, the amendment of the User Profile and a location aware UI.

\subsection{Service Usage}

By mining the Usage Log, it is possible to ascertain the number of times a service has been used. If it transpires that throughout the duration of the HoD's usage a service is never used, the UI and User Profile can be modified and thus personalised to reflect that.

A simple rule based algorithm was applied in order to ascertain the number of times each service was activated.

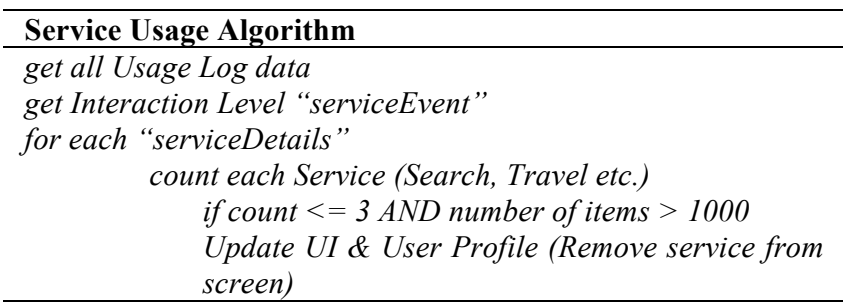

As a result of this personalisation of the User Profile, the UI of the application can adapt and remove the unused service completely from the screen. The service is still available through sub-menus, however, it is no longer prominent on the main homescreen.

\subsection{Storage of Favourites}

Any regular or commonly searched for query terms can be recorded and stored for quick retrieval within specific services. For example, if the user regularly searches for "Restaurants" within the Travel service, after a pre-defined, however, customisable, number of requests for this query, a 'Favourites' table is populated with this search query and its related service. When the user enters the Travel service screen, they are presented with the option to search for "Restaurants" without the need to type the query in again. This same principle is applied to other services such as 'Search' shown in Figure 4.

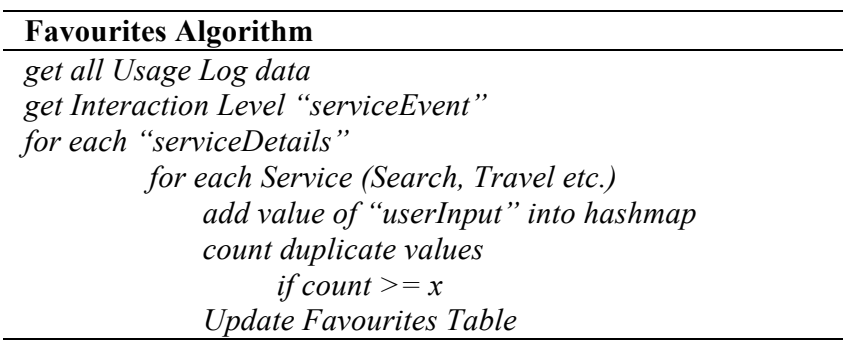

This capability will improve the user experience within the application by decreasing the cognitive load and amount of interactions the user has to perform. This will benefit the target cohort in terms of the reduced burden on the dexterity of their fingers when typing on a small touch screen keyboard [17] and in turn reduces cognitive load. 


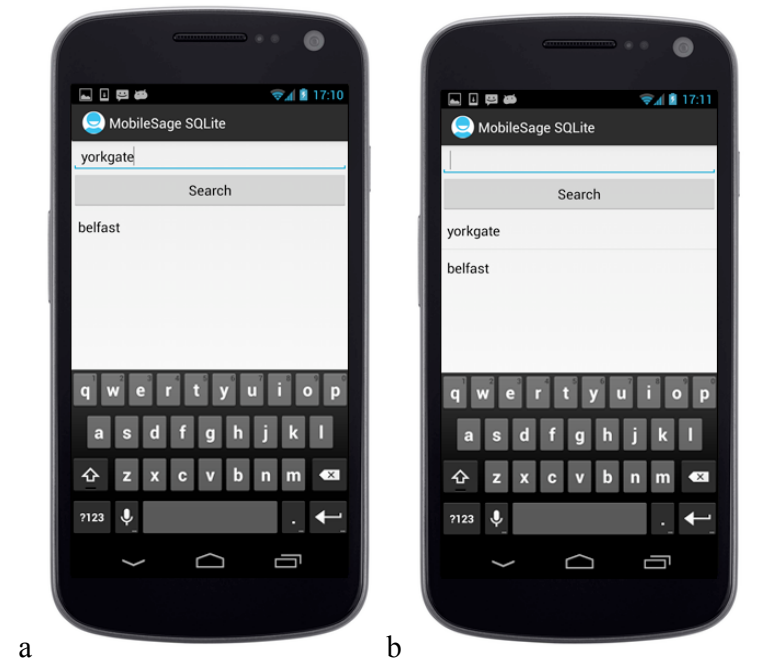

Figure 4. a) User Interface of the Search services showing the onscreen keyboard with a list of most searched for queries and b) showing the latest "favourite" search query added to the list

\subsection{Modification of Requests Based on Device Capabilities}

In order to offer the best user experience and extend the capabilities of the device, the current signal strength is queried when a request is being made to the CMS. If the user's preferred feedback mode is video and their signal strength or battery level is below a certain threshold, the system can prompt the user to modify the feedback mode to something more suitable to the current bandwidth or device capability.

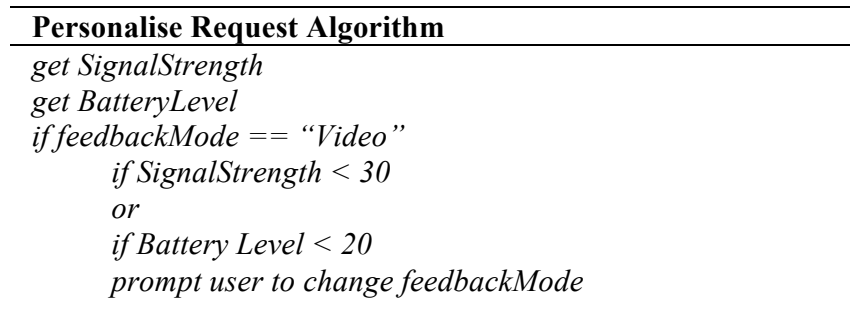

Given that the application is designed for use in a number of contexts it is important that the best possible experience is provided in all contexts. This capability will monitor the context of the device hardware and adapt the content requests in order to provide the best user experience and performance.

\subsection{Amendment of the User Profile}

Personalisation of the system comes primarily from the User Profile. This profile is established and populated during the initialisation phase of the HoD usage. During the usage life of the application it is likely that the user's preferences and usage patterns will change as they become more familiar with the technology and content. In order to provide up to date personalisation, the Usage Log is mined for specific patterns of usage relating to server communications. For example, if the user's preferred feedback mode is video, however, when they use specific functions they often change the requested feedback mode on-the-fly to audio, then the Data Mining component can identify these events and amend the User Profile to match the new feedback mode, meaning the user no longer needs to change this option on-the-fly.

Other patterns can be extracted from the Usage Log to provide adaptation, for example if the audio or video content's volume is too low, this is identified in the Usage Log by a serverResponse followed by a deviceEvent of KEYCODE_VOLUME_UP. In this instance the device's volume can be automatically increased to its maximum level as a request is being made. If it transpires that the device volume is still not sufficient enough for the user's capabilities, the feedback mode can be modified to Text, or a request can be made to the CMS to increase the volume level of the Video content on the server side, before it is sent to the user.

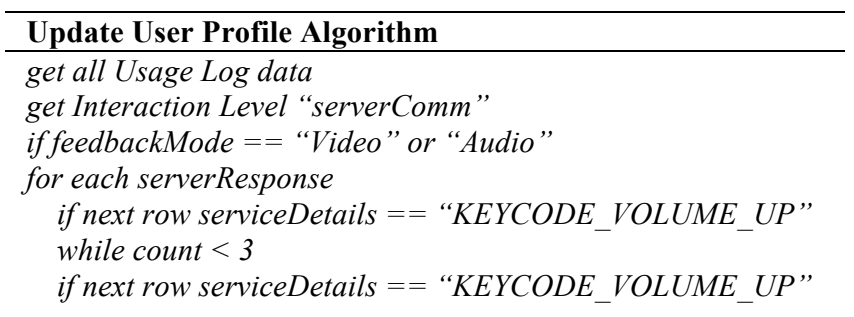

\section{Update User Profile Volume Parameter}

This capability allows the personalisation of the User Profile, which in turn is used to inform the data mining component and adapt the application UI.

\subsection{Location Aware UI}

As each event stored in the Usage Log contains a GPS location it is possible to cluster the locations in which specific services were used and therefore determine the centroids and generate proximity alerts. In order to achieve this, the Usage Log is queried for all service interactions and retrieves their locations. With these locations containing two coordinates and being able to be stored in a 2-dimentional vector, a $k$ Means [18] clustering algorithm was chosen as a suitable approach to generate centroids. The number of clusters generated depends on the number of locations within the dataset, with the total number of locations being divided by 10 , essentially resulting in clusters containing at least 10 locations, as presented in Figure 5.

There are, however, some weaknesses of using kMeans, namely, with fewer samples of data inaccurate clustering can occur. Nevertheless, the more the user uses the application and generates data, the more accurate the clustering will be. For some uses the resulting circular clusters will not suffice. As we are using Google Android's Proximity Alert API ${ }^{1}$ that is also based on distance, the boundaries created will match the resulting centroids.

\footnotetext{
${ }^{1}$ Android Proximity Alerts are part of the LocationManager class. http://developer.android.com/reference/android/location/Locatio nManager.html
} 


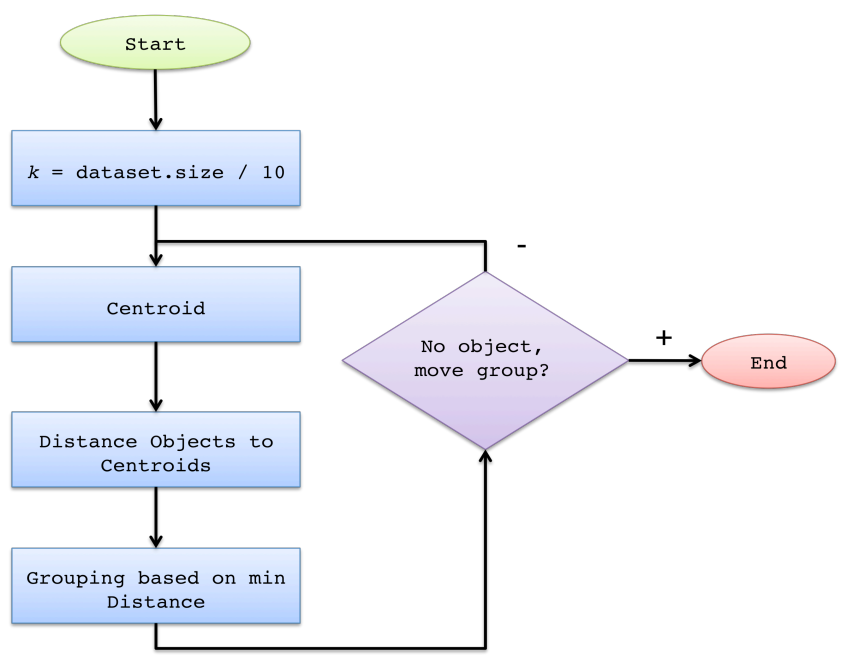

Figure 5. Flow of information within kMeans Algorithm for extraction of centroids.

The generated centroids represent the mean location of each cluster. They are stored in another table that contains the service name, latitude and longitude of the centroid as presented in Figure 6 . The centroids table is repopulated each time the data mining component is run. Using the Proximity Alert API, the application can listen to the current location of the user and determine if they are in a predefined range of the centroids. If this is the case, the UI is modified to reflect that the user is in a location that a specific service is commonly used.

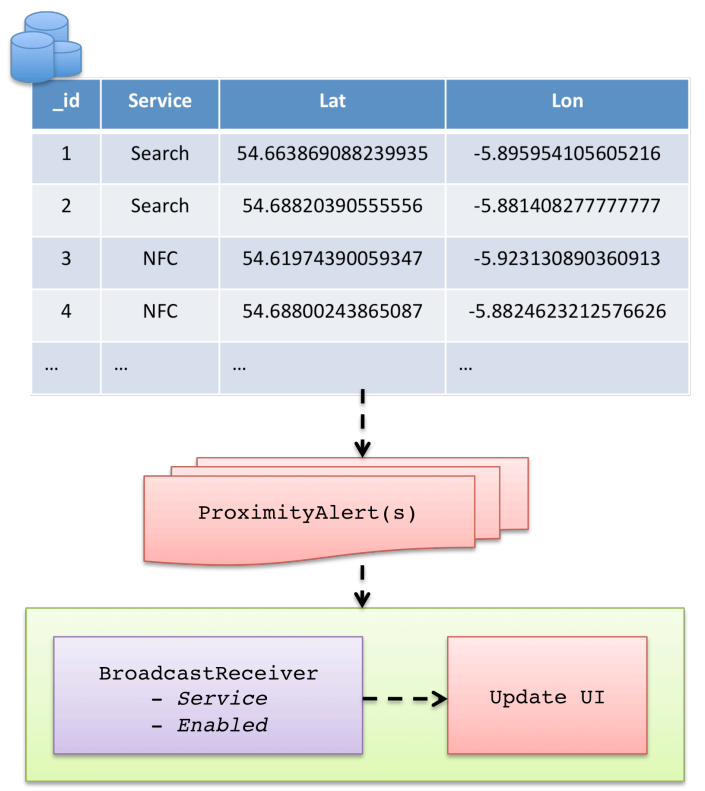

Figure 6. The flow of information from the centroid table through the Proximity Alert API and subsequent Broadcast Receivers to the Updated UI.

\section{TESTING AND EVALUATION}

In this section we outline the methods used to evaluate the collection of usage data and the mining of that data for usage patterns.

\subsection{Experiment Setup}

To test the features of the Data Mining component, data was collected with a test application developed to mimic the envisaged MobileSage prototype. This application collected the usage data based on the structure outlined in Section 3.1. No requests were made to an external CMS and no responses were received. All server requests and responses were simulated, nevertheless the serverComms (Level 4) event types were recorded in the Usage Log.

The proof of concept application was given to three healthy adults, two female (both aged 24) and one male (age 27) to collect data within their home and work environments. The application was used as often as possible in a number of different locations around a city area in order to build up a suitable dataset to be mined. The usage of the application varied and did not, knowingly, follow any particular pattern. The only predefined actions were the accessing of specific services in a specific location in order to test the location mining and searching for the same search query for a number of times, to test the "Favourites" component (Section 6.2).

The application was deployed on three NFC enabled Smartphones: two Nexus $\mathrm{S}$ and one Galaxy Nexus. The participants were requested to access services of their choice as they travelled around between different locations.. When any interaction with the application takes place it is recorded in the Usage Log with the parameters outlined above. This will build a dataset of the user's interaction patterns that will be mined to personalise and adapt the application.

The kMeans algorithm employed within this solution was compared to that of the WEKA Data Mining system's Simple kMeans algorithm [19]. This was employed in order to validate the effectiveness of the developed algorithm.

Figure 7 shows the resulting UI of the proof of concept application when the user is in location where the Search service is the most commonly used service. It should be noted that at this stage of the work the aesthetics of the UI have not been taken into consideration with users. The main focus of the evaluation related to the performance of the data capture and storage, and data mining components, especially the adaptation of the application UI based on the user's current location context.

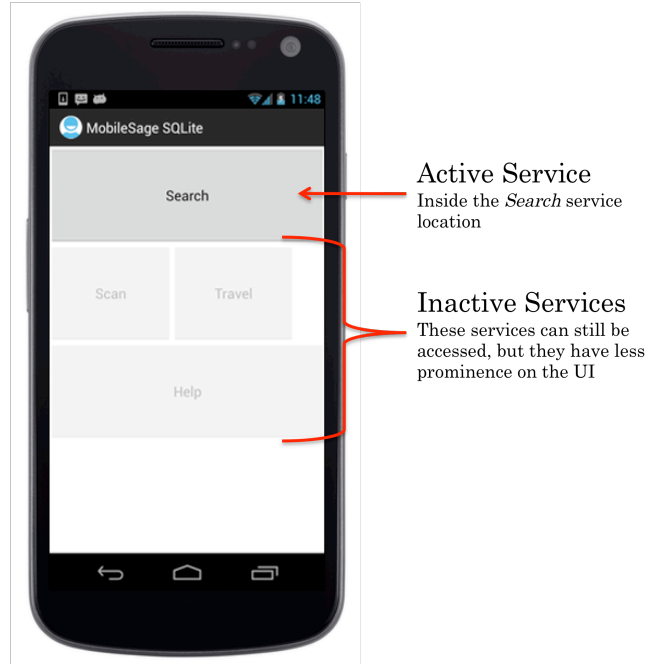

Figure 7. Screenshot of the adapted UI when the user enters a designated area around a Search centroid. Unused service 
button's alpha values are decreased in order to provide prominence to the 'Active' service button.

In the event the user is in a location where there is no centroid, hence no most used service, all of the services are available until such a time as one is used more than the others. Nevertheless, if a user uses more than one service in the same location, then all of those services will be displayed on the screen.

\subsection{Results and Discussions}

In this Section we present the results of the data mining component of the application. The data, specifically the Location aware UI was compared to the WEKA simpleKMeans algorithm, using the same datasets.

Table 2 outlines the number of usage log events that were recorded by each participant in addition to the total number of service activations, with locations used.

TABLE 2. INFORMATION ON THE PARTICIPANTS OF THE EVALUATION AND THE TOTAL NUMBER OF EVENTS AND SERVICE ACTIVATIONS RECORDED DURING THE EVALUATION PERIOD.

\begin{tabular}{|c|c|c|c|c|c|c|}
\hline User & Sex & $\begin{array}{c}\# \\
\text { events } \\
\text { logged }\end{array}$ & Search & $\begin{array}{c}\text { Scan } \\
\text { (NFC } \\
\text { \& } \\
\text { QR) }\end{array}$ & Travel & $\begin{array}{c}\text { Total \# } \\
\text { service } \\
\text { activations }\end{array}$ \\
\hline 1 & $\mathrm{M}$ & 820 & 55 & 87 & 16 & 158 \\
\hline 2 & $\mathrm{~F}$ & 611 & 33 & 41 & 13 & 87 \\
\hline 3 & $\mathrm{~F}$ & 745 & 77 & 31 & 16 & 124 \\
\hline
\end{tabular}

Figures 8, 9 and 10 show all of the users' service activation locations on a map in addition to the generated centroids from the application kMeans algorithm and WEKA's own algorithm.

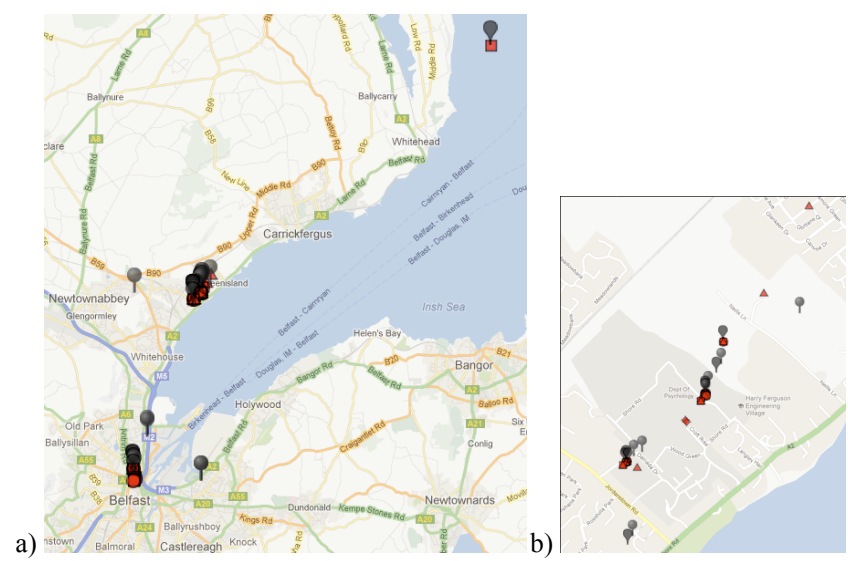

Figure 8. a) All 158 locations recorded by User 1, plotted on a map (RED) with the generated centroids from 1 ) the applications $P$ and 2) WEKA $P$. b) A higher granularity of the comparison.

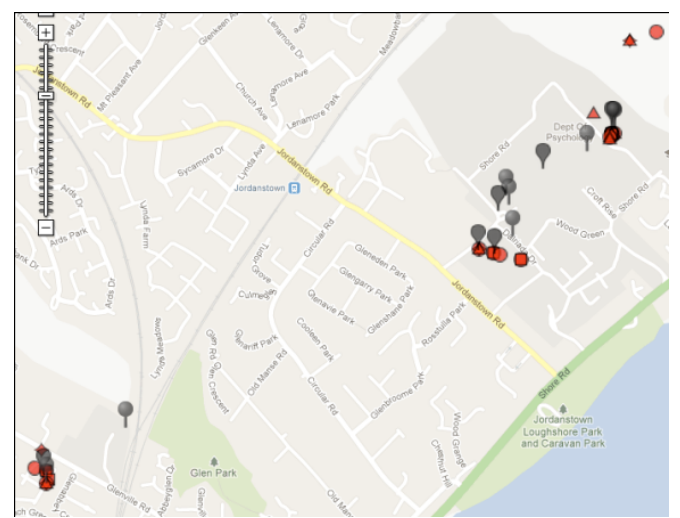

Figure 9. a) All 87 locations recorded by User 2, plotted on a map (RED) with the generated centroids from 1) the applications 9 and 2) WEKA?

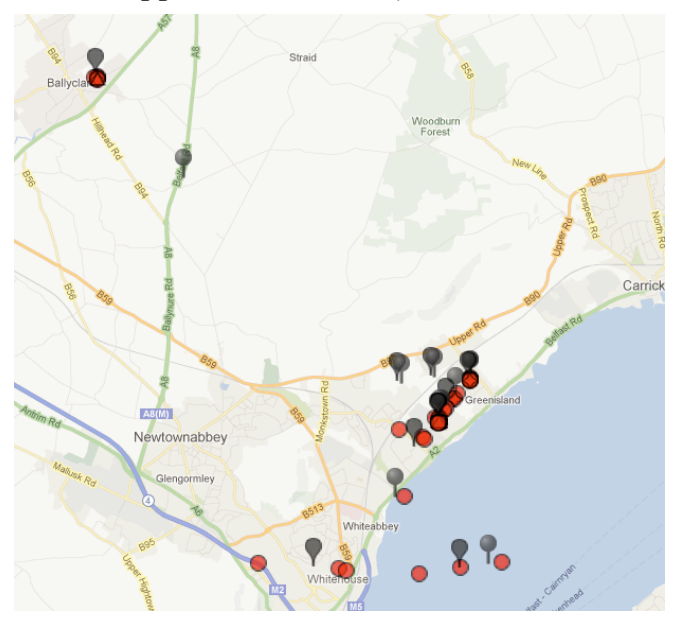

Figure 10. a) All 124 locations recorded by User 3, plotted on a map (RED) with the generated centroids from 1) the applications $P$ and 2) WEKA ?

As we can see from the Figures above, the centroids generated by the application algorithm differ in terms of location to those from the WEKA algorithm. As Table 3 shows, some of the generated centroids from the application algorithm are very close to the centroids generated from the WEKA algorithm.

TABLE 3. GENERATED CENTROIDS FROM BOTH ALGORITHMS OF THE SEARCH SERVICE ACTIVATIONS FROM USER 1.

\begin{tabular}{lll}
\hline Algorithm & \multicolumn{1}{c}{ Latitude } & \multicolumn{1}{c}{ Longitude } \\
\hline App & 54.6882537 & -5.880914833333333 \\
WEKA & 54.6097 & -5.9315 \\
App & 54.6885842 & -5.880739707142857 \\
WEKA & 54.6883 & -5.8809 \\
App & 54.62541692568865 & -5.921402456761713 \\
WEKA & 54.6855 & -5.8866 \\
App & 54.68572237272726 & -5.886109681818181 \\
WEKA & 54.6883 & -5.8809 \\
App & 54.688338607142875 & -5.880916099999999 \\
WEKA & 54.6905 & -5.8796 \\
\hline
\end{tabular}


Despite the slight inaccuracy of some of the generated centroids from the application algorithm, the overall performance of the application was acceptable by all users. The UI adapted based on the user locations after sufficient data was collected. The most searched for queries were also mined and stored in a separate table to be displayed in the UI. Other aspects of the data mining component performed well such as the personalisation of the User Profile and the adaptation of server requests based on device capabilities.

\section{CONCLUSIONS}

In this paper we introduced a framework that can analyse usage data of the Help-on-Demand service to detect preferences and changes of user behaviors, thus providing adaptive personalised services and user interfaces. We have detailed the rule based learning and $\mathrm{k}$-means clustering methods and exemplified the development of use of these methods for usage pattern recognition as well as for content and UI personalisation.

The case studies have demonstrated that the proposed framework and associated data mining methods are working. Though current experiments are based on a simulated dataset, there is no reason why these methods would not work on a real dataset.

At this point, the usage models and collection component have been integrated into the HoD prototype and real usage data have already been collected in the context of the MobileSage project. Future work aims to integrate the data mining component into the HoD and perform usage pattern recognition based on the collected real usage data using the methodologies described within this paper.

\section{ACKNOWLEDGMENTS}

This work is supported by the EU AAL MobileSage project. The authors gratefully acknowledge the contributions from all members of the MobileSage consortium.

\section{REFERENCES}

[1] WHO Europe: http://www.euro.who.int/en/what-wedo/health-topics/Life-stages/healthyageing/news/news/2012/01/ageing-and-health-in-focus-in2012. (Accessed: January 2013)

[2] Doukas, C., Metsis, V., Becker, E., Le, Z., Makedon, F., Maglogiannis, I. 2011. Digital cities of the future: Extending (a) home assistive technologies. Telematics and Informatics 28(3), 176-190.

[3] Beach A, Gartrell M, Xing X et al., 2010. Fusing mobile, sensor, and social data to fully enable context-aware computing. In Proceedings of the Eleventh Workshop on Mobile Computing Systems \& Applications (HotMobile '10). ACM

[4] Remmers, H. 2010. Environments for ageing, assistive technology and self-determination: ethical perspectives. Informatics for health \& social care, 35(3-4), 200-210.

[5] Dey, A.K. 2001 Understanding and using context. Personal and Ubiquitous Computing 5(1), 4-7
[6] Miskelly, F. 2005. Electronic tracking of patients with dementia and wandering using mobile phone technology. Age and Ageing 34, 497-518

[7] Donnelly, M., Nugent, C., McClean, S., et al. 2010 A Mobile Multimedia Technology to Aid Those with Alzheimer's Disease. IEEE Multimedia 17(2), 42-51

[8] Boulos, M.N.K., SWheeler, S.,, Tavares, C., Jones, R. 2011 How smartphones are changing the face of mobile and participatory healthcare: an overview, with example from eCAALYX. BioMedical Engineering Online 10(24).

[9] Heckmann, D. Schwartz, T. Brandherm, B. et al. 2005. GUMO - The general user model ontology. $10^{\text {th }}$ International User Modeling Conference. Pp 428 - 432.

[10] Sutterer, M. Droegehorn, O. David, D. 2008. UPOS: User Profile ontology with situation-dependent preferences support. $1^{\text {st }}$ International Conference on Advances in Human Computer Interaction. Pp 230-235

[11] Li, G. Zou, H. Yang, F. 2011. User Preference Based Service Personalization Using iXCS. Journal of Information \& Computational Science 8: 12(60821001), 2301-2313.

[12] Montoliu, R., Blom, J., \& Gatica-Perez, D. 2012. Discovering places of interest in everyday life from smartphone data. Multimedia Tools and Applications. doi:10.1007/s11042-011-0982-z

[13] Weiss, G. Lockhart, J. 2012. The impact of personalization on smartphone-based activity recognition. Activity Context Representation: Techniques and Languages 98-104.

[14] Höpken, W., Fuchs, M., Zanker, M., \& Beer, T. 2010. Context-Based Adaptation of Mobile Applications in Tourism. Information Technology \& Tourism, 12(2), 175195. doi:10.3727/109830510X12887971002783

[15] MobileSage Group AAL. MobileSage - Situated Adaptive Guidance for the Mobile Elderly, http://www.mobilesage.eu (Accessed January 2013)

[16] Burns, W. Chen, L. Nugent, C. Donnelly, M. et al. 2012. A Conceptual Framework for Supporting Adaptive Personalized Help-on-Demand Services. AmI 2012, LNAI 7683, pp. 427-432

[17] Hwangbo, H., Yoon, S. H., Jin, B. S., et al. 2012. A Study of Pointing Performance of Elderly Users on Smartphones. International Journal of Human-Computer Interaction. doi:10.1080/10447318.2012.729996

[18] MacQueen, J. 1967. Some methods for classification and analysis of multivariate observations. Fifth Berkeley Symposium on Mathematical Statistics and Probability. Vol. 1 (Univ. of Calif. Press, 1967), 281-297

[19] WEKA SimplekMeans algorithm: http://weka.wikispaces.com/Using+cluster+algorithms (Accessed: January 2013 